\title{
Propiedades Psicométricas de la Escala de Connor-Davidson Resilience (CD- RISC) en Adolescentes Escolarizados Chilenos
}

\section{Psychometric Properties of the Connor-Davidson Resilience Scale (CD-RISC) in Chilean School Adolescents}

\author{
Karina Blanco-Molina ${ }^{1}$, Sonia Salvo-Garrido ${ }^{2}$, Paula Alarcon-Bañares ${ }^{3}$, \\ Ítalo Trizano-Hermosilla ${ }^{4}$ y Sebastián Candia Monsalve ${ }^{5}$
}

\begin{abstract}
Resumen
El objetivo del estudio fue examinar las propiedades psicométricas de la escala CD-RISC en población adolescente chilena. La muestra fue de 520 estudiantes, en un rango etario de 13 a 19 años, pertenecientes a establecimientos escolares de las regiones de La Araucanía y Los Ríos, Chile. Los resultados del análisis factorial confirmatorio determinó una estructura unidimensional mediante un modelo Bifactor, compuesta por un factor general de resiliencia y dos sub dimensiones, con adecuados indicadores de ajuste (CFI=.956; RMSEA=.05). La fiabilidad mediante consistencia interna presentó rangos estadísticamente esperables, con un coeficiente Omega jerárquico de .81. El nivel de invarianza de la escala para las variables sexo y edad fue escalar, no encontrándose diferencias significativas en el factor general de resiliencia. Se obtuvieron evidencias de validez convergente y discriminante. Por tanto, CD-RISC, muestra indicadores psicométricos adecuados para adolescentes chilenos evaluados y se discutieron las limitaciones del presente estudio.
\end{abstract}

Palabras clave: resiliencia, CD-RISC, adolescencia, escala, recursos resilientes

\begin{abstract}
This study aimed to examine the psychometric properties of the CD-RISC scale in the Chilean adolescent population. The sample was 520 students within an age range of 13 to 19 years, belonging to schools in the regions of La Araucanía and Los Ríos, Chile. The confirmatory factor analysis results determined a onedimensional structure using a Bifactor model, composed of a general factor of resilience and two subdimensions, with suitable adjustment indicators (CFI=.956; RMSEA=.05). Reliability through internal consistency showed statistically expected ranges, with a hierarchical Omega coefficient of .81. The level of invariance of the scale for the sex and age variables was scalar, not finding significant differences in resilience's general factor. We did find evidence of convergent and discriminant validity. Therefore, CDRISC showed consistent psychometric indicators for the Chilean adolescents evaluated. Finally, we discuss the limitations of this study.
\end{abstract}

Keywords: resilience, CD-RISC, adolescence, scale, resilient resources

\footnotetext{
${ }^{1}$ Magíster en Psicología Jurídica y Forense, Universidad de La Frontera, Avenida Francisco Salazar 01145, 4780071, Temuco, Chile. Tel.: +56-45-2596690. Correo: karinablancomolina@gmail.com

${ }^{2}$ Dra. en Estadística Multivariante, Universidad de La Frontera, Avenida Francisco Salazar 01145, 4811230, Temuco, Chile. Investigadora del Laboratorio de Investigación en Ciencias Sociales Aplicadas (LICSA). Tel.: +56-045-2325670. Correo: sonia.salvo@ufrontera.cl (Correspondencia).

${ }^{3}$ Dra. en Psicología, Académica Departamento de Psicología, Universidad de La Frontera, Avenida Francisco Salazar 01145, 4811230, Temuco, Chile. Tel.: +56-45-2325608. Correo: paula.alarcon@ufrontera.cl

${ }^{4}$ Dr. en Psicología, Académico Departamento de Psicología, Universidad de La Frontera, Avenida Francisco Salazar 01145, Temuco, Chile. +56-45- 2325612. Código postal: 4811230, Correo: italo.trizano@ufrontera.cl

${ }^{5}$ Magíster en Psicología Jurídica y Forense, Universidad de La Frontera. Avenida Francisco Salazar 01145, 4811230, Temuco, Chile. Tel.: +56-45-2596690. Correo: scandiamonsalve@gmail.com
}

Revista Iberoamericana de Diagnóstico y Evaluación - e Avaliação Psicológica. RIDEP · No57 · Vol.4 · 163-176 · 2020

ISSN: 1135-3848 print /2183-6051online 


\section{Introducción}

El constructo de resiliencia ha sido estudiado y desarrollado por diferentes disciplinas, sin lograr todavía un consenso en su definición (Farsen et al., 2017). La evidencia acumulada, permite reconocer características como el dinamismo y multidimensionalidad del constructo (Aburn et al., 2016; Roque Herńandez et al., 2009), determinando que se construye por diferentes aspectos: la existencia de un estresor, el poseer la capacidad de flexibilidad del sujeto (Aburn et al., 2016; Luthar et al., 2000), la activación de mecanismos y factores psicosociales que permitan la adaptación positiva (Barcelata, 2015; Saavedra, 2010), favoreciendo en su conjunto, el bienestar psicológico (Aburn et al., 2016; Dray et al., 2017). Actualmente se reconoce la resiliencia, como la capacidad de sobreponerse a situaciones adversas y ajustarse positivamente en el entorno (Farsen et al., 2017).

El constructo teórico de resiliencia, se desarrolla principalmente en cuatro etapas de evolución (Pinto-Cortez, 2014), desde los años 70' con la exposición de factores protectores y riesgo como mediadores del proceso resiliente, y 20 años después el tema se centra en el cómo se adquieren los recursos y mecanismos resilientes (Saavedra, 2010); todo esto, permitió un tercer avance desde la intervención del proceso resiliente y la generación de instrumentos de medida a favor de la evaluación del constructo, donde algunos de los precursores fueron Connor y Davidson (2003); Freiberg, Stover, de la Iglesia y Fernández (2013) y Liebenberg, Ungar y van de Vijver (2012) y, finalmente el avance de la investigación permite incorporar la perspectiva de la neurociencia a los procesos resilientes, dando paso a una cuarta etapa de su complejización (Barudy, 2015).

Actualmente los estudios en resiliencia, se centran en niños y adolescentes con alta exposición a factores de riesgo, su adaptación positiva, la recuperación frente a eventos traumáticos y el cómo se logra mantener la funcionalidad ante la exposición prolongada a eventos estresantes (Barcelata, 2015).

Estudios recientes sobre bienestar psicológico y grado de felicidad de los adolescentes, han demostrado el rol de los recursos resilientes y el autoconcepto, para lograr resultados educativos favorables (Rodríguez-Fernández et al., 2016a), así como, una mayor implicación e integración a nivel conductual, emocional y cognitivo, con su comunidad educativa (Salvo-Garrido, San Martín, et al., 2020). Los adolescentes con mayores recursos resilientes, parecieran invertir mayor tiempo en las tareas de aprendizaje, siendo más participativos y con mejor rendimiento académico que los que poseen menores recursos en resiliencia (Rodríguez-Fernández et al., 2016b; Rodríquez-Fernández et al., 2018; Salvo-Garrido, Miranda-Vargas, et al., 2020).

Por su parte, Villalta, Delgado, Escurra y Torres (2017) enfatizan el rol de las variables socioculturales al estudiar la relación entre rendimiento escolar y resiliencia. Las dimensiones identificadas por los instrumentos pueden comportarse diferente en diversas culturas, según las condiciones de adversidad, el significado que le asignen los adolescentes y su contexto social.

De igual manera, se ha encontrado una relación entre la presencia de expectativas educativas, asistencia a la educación preescolar y recursos resilientes, especialmente, por la probable asociación entre la importancia de iniciar tempranamente la educación (pre-escolar) y las mayores expectativas familiares, junto a la preocupación por un buen rendimiento académico (Bravo-Sanzana, Salvo-Garrido, Mieres, Mansilla, $\&$ Hederich, 2017). Se ha descrito que las altas expectativas educativas de los estudiantes, sumada a un clima social con bajos indicadores de bullying y violencia escolar, reforzarían la resiliencia socioeducativa (Acevedo \& Restrepo, 2012; Alarcón et al., 2012; Salvo-Garrido, San Martín, et al., 2020).

En síntesis, la evidencia revisada, pone el acento en los componentes dinámicos de los recursos resilientes en la adolescencia, enlazando los factores internos/personales con los externos/contextuales, de la comunidad escolar. Por tanto, resulta lógico mejorar las mediciones que permitan detectar y promover resiliencia, especialmente en periodos críticos del desarrollo humano, como lo es, la adolescencia (Hodder et al., 2017). Para ello, se requiere contar con instrumentos que cuenten con evidencias de validez en la población estudiada, considerando las diversidades socioculturales, y la versatilidad de las respuestas que pueden presentar los 
adolescentes para lograr adaptaciones positivas (Lemos et al., 2016; Luthar \& Einsenberg, 2017).

La presente investigación se desarrolla en la región latinoamericana particularmente en Chile, país donde la adherencia al sistema escolar ha sufrido un importante decremento, el 5.9\% de los adolescentes entre 14 a 18 años deserta, y esto se eleva significativamente en el grupo de inmigrantes, a $19.9 \%$, acentuándose su condición de exclusión social (Bonomelli et al., 2020).

Lo anterior fundamenta la necesidad de desarrollar nuevos programas de intervención focalizados en resiliencia, complementarios a la formación escolar, y que permitan potenciar los recursos para mejorar resultados de aprendizaje, e incrementar oportunidades de integración social a través de respuestas más resilientes para esta población. Para desarrollar y evaluar el impacto de los programas se requieren instrumentos pertinentes culturalmente y validados en dicha la población.

\section{Evaluación de Resiliencia}

Las medidas e indicadores de resiliencia han ido evolucionando a la par con los avances en la comprensión del constructo, existiendo en la actualidad una amplia discusión y análisis sobre las mejores herramientas para detectar indicadores de resiliencia (Jones, 2019).

Para conocer el desarrollo de instrumentos que miden resiliencia, un buen punto de partida, es el meta-análisis de Windle, Bennett y Noyes, (2011), que examina 15 escalas de resiliencia, concluyendo para la década estudiada (19892009) que de los 15 instrumentos revisados ninguno contaba con la evidencia de un "Gold standard" psicométrico. A pesar de ello, la revisión jerarquiza tres escalas que miden recursos personales activos en adultos, con adecuados niveles de fiabilidad y validez; The ConnorDavidson Resilience Scale CD-RISC (Connor \& Davidson, 2003), The Resilience Scale for Adults RSEA (Friborg et al., 2003) y la Escala de resiliencia $R S-14$ de (Wagnild \& Young, 1993). No obstante, Windle, Bennett, \& Noyes, (2011), proponen que las variables contextuales y culturales pueden jugar un rol importante en la variabilidad de las mediciones de resiliencia, resaltando instrumentos que la examinan desde múltiples niveles de medición como lo es Child and Youth Resilience Measure, CYRM-28 (Ungar \& Liebenberg, 2011), la Escala de Resiliencia de la Encuesta de Niños Saludables de California, la Resilience Scale for Adolescents, READ (Hjemdal et al., 2006) y el Youth Resiliency: Assessing Developmental Strengths YR: ADS (Donnon \& Hammond, 2007).

Posteriormente, Cosco et al. (2016) a través de una nueva revisión sistemática de 470 artículos sobre resiliencia, reitera el reconocimiento a la escala CD- RISC ( 25 ítems) y CD RISC abreviada (10 ítems), como uno de los instrumentos que acumulan evidencia empírica en diferentes grupos etarios y poblaciones. El CD-RISC, ha sido estudiado en población adulta general, universitaria, clínica y adolescente, con reportes de la fiabilidad por consistencia interna satisfactorios, oscilando entre alpha .88 y .98 .

Esta escala fue desarrollada por Connor \& Davidson (2003) quienes identificaron inicialmente 5 dimensiones que se concentran principalmente en recursos personales, estos son: Factor 1: Competencia personal, altos estándares y tenacidad; Factor 2: Confianza en la intuición, tolerancia a los efectos negativos y fortaleza frente al estrés; Factor 3: Aceptación positiva de los cambios y relaciones seguras; Factor 4: Control; y Factor 5: Influencia espiritual.

Los reportes en evidencias de validez en estructura interna para el CD-RISC, varían en diferentes poblaciones, donde solo algunos estudios han corroborado la estructura factorial de cinco factores propuestos por los autores (Serrano et al., 2012). Estudios en población adulta expuestas a alto estrés; enfermeras de cuidados intensivos en EEUU (Mealer et al., 2016), en soldados en China (Xie et al., 2016) y en emprendedores españoles (Manzano-García \& Ayala Calvo, 2013), coinciden en soluciones de tres factores.

$\mathrm{Al}$ examinar estas soluciones factoriales, se observan correlaciones entre factores, lo que permitiría hipotetizar la existencia de un factor general no identificado. Ponce (2015) al estudiar y comparar dos muestras de estudiantes universitarios chilenos y españoles, se plantéa la hipótesis de unidimensionalidad y somete a verificación un modelo bifactor, constatando la invarianza factorial para un factor global de resiliencia, pero las subdimensiones específicas resultan inestables en las dos muestras. Esta 
evidencia de unidimensionalidad coincide tambien con otros estudios reportados en población española (Arias González et al., 2015; GarcíaLeón et al., 2019).

En síntesis, la amplia evidencia acumulada para el CD-RISC, presenta a esta escala como una de las herramientas más usadas y adaptadas para medir resiliencia en diferentes poblaciones, coincidiendo los autores en reportar adecuados indicadores de fiabilidad, en diversos grupos etarios, clínicos y normativos. Esta evidencia permite seleccionar al CD-RISC para examinar sus propiedades psicométricas en población adolescente en Chile.

Sin embargo, el reporte de evidencias de validez en estructura interna del CD-RISC, no se presenta con claridad en torno a una unidimensionalidad vs multidimensionalidad en su estructura factorial. Esta divergencia puede tener valor en dos direcciones, la primera de ellas se relaciona con la complejidad y dinamismo del constructo de resiliencia, así como, el impacto de las variables socio-culturales en dimensiones de los diferentes grupos estudiados; y la segunda dirección no menos importante, es la fragilidad o menor robustez que podría presentar la prueba, en relación a la estructura factorial y por tanto, requiere una mayor investigación y revisión de los alcances del constructo que mide esta prueba (Abad et al., 2011).

Considerando estos antecedentes, el presente estudio tuvo por objetivo examinar las propiedades psicométricas del instrumento CDRISC en adolescentes chilenos que cursan la educación secundaria (13 a 19 años), no expuestos a experiencias especialmente estresantes. Para conocer evidencia de validez externa, se seleccionó la escala diseñada para adolescentes que examina la resiliencia en múltiples niveles, Child and Youth Resilience Measure CYRM-28 (Ungar \& Liebenberg, 2011).

\section{Método}

\section{Participantes}

La población definida para este estudio correspondió a estudiantes adolescentes de las comunas de Temuco, Chol Chol y Nueva Imperial, Región de La Araucanía y de la comuna de Lanco, Región de Los Ríos, ubicadas al sur de
Chile. La selección de los participantes se realizó mediante un muestreo no probabilístico por conveniencia. La muestra quedó compuesta por 576 adolescentes de educación secundaria pertenecientes a cuatro establecimientos particulares subvencionados ubicados en las regiones de La Araucanía (87\%) y de Los Ríos (13\%). Considerando solo los protocolos válidos para el estudio la muestra quedó constituida por 520 sujetos. El rango etario varía entre los 13 y 19 años, con una media de 16.2 años (DS=1.4). El $31.1 \%$ de los sujetos tenía edades comprendidas entre 13 y 15 años y el $68.8 \%$ entre 16 y 19 años. La mayoría fueron hombres (58.1\%), el $64 \%$ declaró pertenecer a la zona de residencia urbana.

Respecto de la situación familiar y económica auto reportada, el $56.9 \%$ vive con ambos padres y posee un ingreso promedio mensual entre 150,000 a 300,000 pesos chilenos (aproximadamente entre 223.95 USD a 447.90 USD).

\section{Instrumentos}

The Connor-Davidson Resilience Scale, CD-RISC (Connor \& Davidson, 2003). Escala compuesta por 25 ítems y cinco factores (persistencia / tenacidad y fuerte sentido de la autoeficacia, emocional y cognitivo, adaptabilidad/capacidad para recuperarse, control/ significado y significado) en su versión original. Los sujetos deben indicar hasta qué punto cada una de las afirmaciones han sido verdaderas en su caso durante el último mes en una escala tipo Likert con cinco opciones de respuesta ("en absoluto", "rara vez", "a veces", "a menudo" y "casi siempre"), puntuados por 0 ("en absoluto") a 4 ("casi siempre"). La escala posee un rango de 0 y 100 puntos, a mayor puntuación indica mayor nivel de resiliencia. Presenta criterios de confiabilidad interna aceptables con alfa de Cronbach de .89 (Connor \& Davidson, 2003). Se empleó la versión en español (Bobes et al., 2001, 2008) con algunas adaptaciones lingüística que permitiesen una mejor comprensión en población adolescente chilena.

Escala de autoreporte Child and Youth Resilience Measure (CYRM-28). La escala CYRM-28 fue creada por Ungar \& Liebenberg (2011) y está compuesta por 28 ítems en formato de respuesta tipo Likert de 1 a 5 (1=para nada, $2=$ =un poco, $3=$ =algo, $4=$ bastante, y $5=$ mucho) y tres 
factores. Factor 1: Individual (habilidades personales, apoyo de los compañeros y habilidades sociales); Factor 2: Relación con cuidador (cuidado físico y cuidado psicológico) y Factor 3: Contexto (espiritual, educacional y cultural). La consistencia interna fue de .82 (alfa de Cronbach) en población canadiense e indicadores más elevados en investigaciones actualizadas (Artuch, 2014). Ruiz Fernández, Gómez-Vela, Fernández Pulido y Badia Corbella (2014) reportaron un alfa de Cronbach de 90. Para esta investigación se utilizó la versión adaptada y validada por Candia y Blanco (2019) compuesta por 26 ítems en población adolescente chilena, que midió de manera consistente y confiable un constructo unidimensional de resiliencia, mediante el modelo de estructura bifactorial, con los tres sub-factores del modelo original: Factor individual, 11 ítems; Factor relación con cuidadores, 5 ítems y Factor contextual, 10 ítems. La fiabilidad por consistencia interna, medida a través del coeficiente omega de McDonald presentó niveles aceptables, tanto para el factor general, .93, como para los tres factores específicos, $.85, .88$ y .81 , respectivamente.

\section{Procedimiento}

Esta investigación es de naturaleza instrumental, pues se evaluaron parámetros estadísticos para justificar la calidad métrica del instrumento (Ato \& López, 2013).

Como la escala CD-RISC no presenta evidencias de validación en el contexto chileno, los ítems de la escala se sometieron a revisión de pertinencia lingüística por dos jueces expertos y cuatro grupos focales conformado por ocho adolescentes escolarizados con edades entre los 14 y 19 años, diferenciado por sexo, para asegurar la correcta comprensión de los ítems. El tiempo de respuesta de la escala fue de 11 minutos y luego se desarrolló las reflexiones asociadas a la escala. Para las conclusiones se contó con la participación de los dos jueces expertos, donde se modificaron lingüísticamente los ítems 2, 3, 12, 13, 18, 20, 22 y 24 (Tabla 1). Además, se realizaron cambios en las opciones de respuesta de la escala tipo Likert, a saber: ( $0=$ para nada, $1=$ un poco, $2=$ algo, 3=bastante, y 4=mucho) por lo sugerido por los adolescentes que participaron en los grupos focales, argumentando mayor capacidad para entender esta clasificación para contestar el instrumento.

Finalmente, se contactó a las direcciones de cuatro establecimientos educacionales, se socializó el objetivo de la investigación y se solicitó su colaboración. Se hizo entrega de consentimientos (directivos de los establecimientos, padres o adultos responsables de los adolescentes) y asentimiento de los adolescentes donde se expuso la voluntariedad y el resguardo de la confidencialidad de la información entregada considerando aspectos éticos. La toma de muestra se realizó a través de la aplicación de los instrumentos en horarios de clases y de manera colectiva (45 minutos aproximadamente). Se llevó a cabo en un periodo de seis meses, junio a noviembre de 2017.

\section{Análisis de datos}

Para evaluar la estructura factorial de la escala se realizó un procedimiento de validación cruzada evaluando un modelo Bifactor, dividendo la muestra en dos mitades aleatorias de 253 y 267 estudiantes. Con la primera mitad de la muestra se realizó un análisis factorial exploratorio (AFE) mediante el programa FACTOR versión 10.10.03, (Lorenzo-Seva \& Ferrando, 2020). Para la implementación del AFE se utilizó la matriz de correlaciones policóricas (Freiberg et al., 2013), para estimar el número de factores se utilizó el análisis paralelo en su implementación óptima con 500 matrices de correlación aleatorias (Timmerman \& Lorenzo-Seva, 2011), el método de extracción robusto "Minimun Rank Factor Analysis" (Ten Berge \& Kiers, 1991) y la aplicación de un modelo Bifactor (Reise, 2012), que permite evaluar el grado de unidimensionalidad de la escala agrupando la variabilidad de los ítems en torno a un factor común y permitiendo la existencia de factores específicos de algunos subconjuntos de ítems.

Con la segunda mitad de la muestra $(n=267)$, se realizó un análisis factorial confirmatorio (AFC) utilizando el programa MPLUS versión 8.4 (Muthen \& Muthen, 2019) y el método de mínimos cuadrados ponderados con media y la varianza ajustada (WLSMV) con la matriz de correlaciones policóricas (Finney, S.J; DiStefano, 2006).

Posteriormente, con la muestra total, se realizaron tres modelos confirmatorios, 
considerando en primer lugar, el modelo Bifactor y los dos modelos teóricos propuestos en la literatura: uno de cinco factores y otro de tres factores correlacionados.

En general, los índices de ajuste fueron valorados según puntos de corte conocidos: CFI>.90 (Marsh et al., 2004), límite superior del intervalo de confianza al 90\% del RMSEA<.10 (West et al., 2012) y "Weighted Root Mean Square Residual", WRMR $<1$ o "Standarized Root Mean Square Residual", SRM $<.08$ son considerados un buen ajuste (Kline, 2005; Yu \& Muthen, 2002). Se recabó evidencia respecto a la presencia del Factor General (FG) mediante diversos indicadores, como Omega, omega jerárquico general, wh (TrizanoHermosilla \& Alvarado, 2016; Zinbarg, 2006), valores mayores a .75 indican predominio de un único FG (Reise et al., 2012); la varianza común explicada (ECV), porcentaje de correlaciones no contaminadas (PUC) y porcentaje de varianza relativa (PRV) (Bonifay et al., 2015; Brunner et al., 2012; Reise, 2012; Rios \& Wells, 2014). Valores de ECV >.70 y PUC >.70 indican que el sesgo relativo será leve y la variación común puede considerarse esencialmente unidimensional (Rodriguez et al., 2015). Si el PUC<.80, la ECV (FG) $>.60$ y el $\omega \mathrm{h}>.70$ sugieren que la presencia de alguna multidimensionalidad no es lo suficientemente severa como para descalificar la interpretación del instrumento como principalmente unidimensional (Reise et al., 2012). Para los factores específicos, se estimaron los correspondientes omega jerárquico de la subescala, $\omega$ hs (Zinbarg, 2006). Si $\omega$ hs $\geq .30$ (Smits et al., 2015), se considera que poseen interpretación sustantiva al margen del FG.

Con respecto al PRV de las subescalas, si es menor al $50 \%$, menos del $50 \%$ de la varianza confiable en esa puntuación de la subescala se debe a su factor específico, significa que la puntuación de la subescala tiene un valor agregado cuestionable más allá de la puntuación total. Valores superiores al $75 \%$ indican fuerte evidencia en favor de usar score de alguna subescala (Li et al., 2016). Misma interpretación se realiza con respecto al PRV del FG. PRV $>75 \%$ indican que al menos el $75 \%$ de la varianza confiable en la puntuación total se debe al FG. Además, se calculó la replicabilidad de constructo, mediante el coeficiente $\mathrm{H}$, donde valores mayores a .80 sugieren una variable latente bien definida, siendo más probable que sea estable entre los estudios, en tanto, con valores menores o iguales a .80 la variable latente es menos estable entre diferentes estudios (Ferrando \& Lorenzo-seva, 2017).

Para estimar el grado de estabilidad psicométrica de la Escala, se realizaron una serie de procedimientos evaluando el grado de invarianza factorial mediante el método de WLSMV para las variables sexo y edad dicotomizada (13-15 años y 16-19 años), considerando los siguientes modelos: Invarianza configuracional, igual número de factores, e Invarianza escalar, igualdad de intersecciones (Vandenberg \& Lance, 2000). No fue posible estimar el modelo de invarianza métrica por separado debido a la generación de problemas de identificación, sugiriéndose pasar directamente del modelo de configuración al escalar (Muthen, 2013). Para comparar los modelos de invarianza se utilizó, además de la diferencia de Chi-cuadrado mediante DIFTEST, las diferencias en CFI $(\triangle \mathrm{CFI})$ y $\triangle$ RMSEA, considerando un valor aceptable para mantener la hipótesis de equivalencia entre los modelos menor a .01 para $\Delta$ CFI y .015 para $\triangle$ RMSE (Kueh et al., 2018).

Con el fin de explorar la evidencia de validez de la escala CD-RISC se analizó la correlación de Pearson entre los scores estimados de los factores generales de resiliencia de las escalas CD-RICS y CYRM-28 en su versión adaptada en adolescentes chilenos. La validez discriminante del FG la escala de resiliencia del CD-RISC se analizó con la variable expectativa de vida extraída de la ficha sociodemográfica, donde los participantes seleccionaron una de las cuatro alternativas (no tengo expectativas, trabajar, estudiar carrera técnica, estudiar carrera universitaria), a través de un ANOVA con pruebas post hoc de Bonferroni, supuesto de homogeneidad de varianza con prueba de Levene, medida del tamaño de efecto global con Eta cuadrado parcial y potencia observada. Estos análisis se realizaron utilizando el software SPSS versión 20.

\section{Resultados}

\section{Evidencias de Validez de estructura interna}

Previo al análisis de la dimensionalidad, se estudió la adecuación de los datos para la realización 
Tabla 1. Indicadores de Bondad de ajuste de los modelos

\begin{tabular}{|c|c|c|c|c|c|c|c|c|}
\hline Modelo & Chi-Cuadrado & Df & p-valor & RMSEA & $\mathrm{P}(\mathrm{RMSE}<=.05)$ & IC90\% & CFI & SRMR \\
\hline 5 Factores & 804.230 & 265 & $<.0001$ & .063 & .000 & {$\left[\begin{array}{ll}0.058 & 0.068\end{array}\right]$} & .927 & .049 \\
\hline 3 Factores & 913.959 & 272 & $<.0001$ & .067 & .000 & {$\left[\begin{array}{ll}0.063 & 0.072\end{array}\right]$} & .913 & .054 \\
\hline Bifactor & 579.191 & 250 & $<.0001$ & .050 & .452 & {$\left[\begin{array}{ll}0.045 & 0.056\end{array}\right]$} & .956 & .040 \\
\hline
\end{tabular}

Tabla 2. Saturaciones factoriales del modelo Bifactor

\begin{tabular}{|c|c|c|c|}
\hline Ítems & FG & F1 & F2 \\
\hline 1. Soy capaz de adaptarme cuando ocurren cambios. & .476 & .341 & \\
\hline $\begin{array}{l}\text { 2. Tengo al menos una relación significativa y segura que me ayuda cuando estoy } \\
\text { estresado (pareja, amigos u otros). }\end{array}$ & .263 & & .064 \\
\hline $\begin{array}{l}\text { 3. Cuando no hay soluciones claras a mis problemas, a veces la "suerte" o "Dios" pueden } \\
\text { ayudarme. }\end{array}$ & .206 & & .255 \\
\hline 4. Puedo enfrentarme a cualquier cosa. & 610 & .185 & \\
\hline $\begin{array}{l}\text { 5. Los éxitos del pasado me dan confianza para enfrentarme con nuevos retos y } \\
\text { dificultades. }\end{array}$ & .752 & - & \\
\hline 6. Intento ver el lado divertido de las cosas cuando me enfrento con problemas. & .358 & .246 & \\
\hline 7. Enfrentarme a las dificultades puede hacerme más fuerte. & 647 & .123 & \\
\hline 8. Tengo tendencia a recuperarme pronto tras enfermedades, heridas u otras privaciones. & .406 & .392 & \\
\hline 9. Bueno o malo, creo que la mayoría de las cosas ocurren por alguna razón. & .322 & & .136 \\
\hline 10. Siempre me esfuerzo sin importar cuál pueda ser el resultado. & .523 & & .449 \\
\hline 11. Creo que puedo lograr mis objetivos, incluso si hay obstáculos. & 651 & & .399 \\
\hline 12. No me doy por vencido a pesar de que las cosas me parezcan que no tienen solución. & .624 & & .317 \\
\hline $\begin{array}{l}\text { 13. Durante los momentos de estrés/crisis (momentos difíciles), sé dónde puedo buscar } \\
\text { ayuda. }\end{array}$ & .428 & & .129 \\
\hline 14. Bajo presión (condiciones de estrés), me centro y pienso claramente. & .488 & .221 & \\
\hline $\begin{array}{l}\text { 15. Prefiero intentar solucionar las cosas por mí mismo, a dejar que otros tomen todas } \\
\text { las decisiones. }\end{array}$ & .463 & .205 & \\
\hline 16. No me desanimo fácilmente con el fracaso. & .417 & .184 & \\
\hline $\begin{array}{l}\text { 17. Creo que soy una persona fuerte cuando me enfrento a los retos y dificultades de la } \\
\text { vida. }\end{array}$ & .783 & .217 & \\
\hline 18. Puedo tomar decisiones difíciles, incluso si afectan a otras personas, si es necesario. & .514 & .308 & \\
\hline $\begin{array}{l}\text { 19. Soy capaz de manejar sentimientos desagradables y dolorosos como tristeza, temor y } \\
\text { enfado. }\end{array}$ & .405 & .486 & \\
\hline $\begin{array}{l}\text { 20. Al enfrentarme a los problemas de la vida, a veces actúo por un } \\
\text { presentimiento (instinto) sin saber por qué. }\end{array}$ & .401 & .159 & \\
\hline 21. Tengo muy claro lo que quiero en la vida. & .472 & & .457 \\
\hline 22. Tengo la sensación que puedo controlar mi vida. & .551 & & .298 \\
\hline 23. Me gustan los retos. & .628 & .069 & \\
\hline $\begin{array}{l}\text { 24. Me esfuerzo y trabajo para conseguir mis objetivos sin importarme las dificultades que } \\
\text { encuentro }\end{array}$ & 637 & & .446 \\
\hline 25. Estoy orgulloso de mis logros. & .437 & & .413 \\
\hline Coeficiente omega total: $\omega$ & .915 & .879 & .840 \\
\hline Coeficiente omega jerárquico: $\omega \mathrm{h}$ & .808 & .130 & .253 \\
\hline Coeficiente omega jerárquico de la subescala: $\omega$ hs & - & .149 & .302 \\
\hline Porcentaje de Varianza Relativa $\left(\omega \mathrm{h} / \omega^{*} 100\right)$ : PRV & 88.3 & 14.8 & 30.1 \\
\hline Porcentaje de Varianza Relativa de la Subescala $(\omega \mathrm{hs} / \omega * 100)$ : PRV ${ }_{\text {sub }}$ & - & 17.0 & 36.0 \\
\hline Varianza común explicada: ECV & .756 & .104 & .140 \\
\hline Replicabilidad de constructo $\mathrm{H}$ & .914 & .514 & .597 \\
\hline PUC & .513 & & \\
\hline
\end{tabular}

de un análisis factorial mediante las pruebas de Kaiser-Meyer-Olkin (KMO) y de esfericidad de Bartlett. Con la primera mitad de la muestra, 253 sujetos, se obtuvo un KMO de .860 (I. de C. al 95\%: .859, .878) y el test de esfericidad de Barlett fue significativo $\left(\chi^{2} \quad(300)=6210.3, \quad p^{<.001)}\right.$, valores que pueden considerarse como buenos.

Al realizar el AFE sobre la escala CD-RISC con la muestra 1, evaluando un modelo Bifactor con un FG y dos factores específicos, los índices de ajuste fueron aceptables con valores de $\mathrm{CFI}=.990, \mathrm{RMSEA}=.032$ (intervalo de confianza
$90 \%$ entre .01 y .05$)$ y RMSR $=.056$. Las saturaciones factoriales del FG variaron entre .190 у .661 y para los factores específicos entre -.252 y .617 para el factor 1 y entre -.351 y .487 para el factor 2 .

Posteriormente, se confirmó esta estructura con la segunda mitad de la muestra, 267 sujetos, a través de un AFC. Los índices de ajuste fueron significativos y satisfactorios: $\chi^{2}(250)=406.074$, $p<.001, \mathrm{CFI}=.962, \mathrm{RMSEA}=.048(90 \%$ IC $.040-$ .057) y SRMR $=.045$. Las saturaciones factoriales del FG variaron entre .229 y .785 y para $\operatorname{los} 7$ 
Tabla 3. Índices de ajuste y comparación entre los modelos de invarianza para sexo y edad

\begin{tabular}{lccccccccccccc}
\hline Variable & Modelo & $\mathrm{c} 2$ & $\mathrm{gl}$ & $\mathrm{p}$ & CFI & RMSEA & $90 \% \mathrm{IC}$ & SRMR & $\Delta \chi 2$ & $\Delta \mathrm{gl}$ & $p$ & $\Delta$ CFI & $\Delta$ RMSEA \\
\hline \multirow{2}{*}{ Sexo } & M0 & 823.906 & 500 & $<.001$ & .956 & .050 & $.044-.056$ & .049 & & & & & \\
& $\mathrm{M} 1$ & 987.252 & 619 & $<.001$ & .950 & .048 & $.042-.053$ & .055 & 215.292 & 119 & $<.001$ & -.006 & -.002 \\
\multirow{2}{*}{ Edad } & M0 & 785.092 & 500 & $<.001$ & .961 & .047 & $.040-.053$ & .047 & & & & & \\
& M1 & 856.368 & 619 & $<.001$ & .968 & .038 & $.032-044$ & .051 & 140.667 & 119 & .0854 & .007 & -.009 \\
\hline
\end{tabular}

factores específicos entre .216 y .509 para el factor 1 y entre -.209 y .579 para el factor 2 .

Una vez determinada la estructura latente, se procedió a comparar el modelo propuesto con los dos modelos teóricos descritos, utilizando la muestra total. En la Tabla 1 se presenta los resultados de los tres modelos de AFC. El modelo Bifactor es el que evidenció mejores índices de bondad de ajuste y el más parsimonioso.

Para el modelo Bifactor, los índices de ajuste fueron significativos y adecuados para el modelo, con valores de $\chi^{2} \quad(250)=579.191, \quad p<.001$, $\mathrm{CFI}=.956$, RMSEA=.050 (intervalo de confianza $90 \%$ entre .045 y .056$)$ y $S R M R=.040$.

La Tabla 2 presenta las cargas factoriales estandarizadas para AFC del modelo Bifactor y los índices de fiabilidad del FG y de las subescalas. Las cargas factoriales estandarizadas para el FG fueron todas estadísticamente significativas $(p<.001)$ y oscilaron entre .206 en el ítem 3 ("Cuando no hay soluciones claras a mis problemas, a veces la "suerte" o "Dios" pueden ayudarme") y .783 en el ítem 17 ("Creo que soy una persona fuerte cuando me enfrento a los retos y dificultades de la vida"). Sólo dos cargas factoriales estandarizadas fueron menores que .3, ítem 2 ("Tengo al menos una relación significativa y segura que me ayuda cuando estoy estresado (pareja, amigos u otros") con un valor de .263 e ítem 3. Por su parte, las saturaciones de los factores específicos variaron entre .064 (ítem 2, factor 2) y .486 (ítems 19, factor 1), mayoritariamente (23 ítems) fueron comparativamente menores que las del FG.

\section{Estimación fiabilidad de la escala}

Una vez determinada la estructura interna de la escala, se evaluó la fiabilidad de los factores, ver Tabla 2. Para el FG de resiliencia se obtuvo un coeficiente omega de .915 , omega jerárquico de .81 y ECV de .756. Estos indicadores evidenciaron adecuados valores de fiabilidad para el FG. El valor de la replicabilidad de constructo,
$\mathrm{H}$, fue de .914, lo que sugiere que el $\mathrm{FG}$ de resiliencia está bien definido, es más probable que sea estable entre los estudios. El valor del PUC fue de .513 , ECV (FG) de $.756 \mathrm{y} \omega \mathrm{h}$ de .801 , lo que evidencia que el instrumento es principalmente unidimensional.

Respecto a la estimación de la fiabilidad para los factores específicos, para el factor 1, se presentó un valor omega jerárquico de .13, en tanto, para el factor 2 un valor de .253. Los coeficientes omega jerárquicos de las subescalas fueron de .149 y .302 , respectivamente. Un valor de $\mathrm{H}$ de .514 para el factor $1 \mathrm{y}$ de .597 para el factor 2. Todos estos valores son considerados bajos, lo que evidencia la fiabilidad del FG de resiliencia.

\section{Evidencia de invarianza factorial}

Al evaluar los modelos de invarianza de configuración y de umbrales (escalar) para la variable sexo y edad, se observaron satisfactorios índices de ajuste para ambos modelos (ver Tabla 3). Al comparar estos modelos se observan diferencias estadísticamente significativas $(p<.001)$ considerando la variable sexo, por lo que se recurre a criterios adicionales para evaluar el grado de invarianza mediante las diferencias de CFI y RMSEA. Con estos criterios se mantiene la hipótesis de equivalencia entre ambos modelos, por lo que se acepta el nivel de invarianza de umbrales. Para la variable edad, no se aprecia diferencias estadísticamente significativas ( $p=.0854$ ), por lo que se mantiene la hipótesis de equivalencia entre ambos modelos, permitiendo la elección del modelo más parsimonioso, en este caso el de invarianza de umbrales.

Una vez determinado el nivel de invarianza, se procedió a comparar las medias latentes (ver Tabla 4), no se observan diferencias estadísticamente significativas para el FG de resiliencia para ambas variables de agrupación. Solo se aprecian diferencias estadísticamente significas en algunos factores específicos, que, sin embargo, no pueden ser interpretados debido a su baja fiabilidad. 
Tabla 4. Medias latentes estimadas para el grupo de hombres (mujeres fijas a 0 ) y de mayor edad (menores son fijadas a 0 )

\begin{tabular}{ccccc}
\hline & & FG & F1 & F2 \\
\hline \multirow{2}{*}{ Sexo } & Media & -.022 & -.593 & -.699 \\
& $p$ & .840 & .000 & .000 \\
\multirow{2}{*}{ Edad } & Media & .125 & .054 & -.341 \\
& $p$ & .220 & .743 & .013 \\
\hline
\end{tabular}

Tabla 5. Medias de la escala CD-RISC de acuerdo al nivel de expectiativa de vida

\begin{tabular}{lcccc}
\hline Nivel de expectativa & N & Media & DS & Post Hoc* \\
\hline No tengo expectativas & 50 & 59.2 & 15.2 & $\mathrm{a}$ \\
Trabajar & 75 & 60.9 & 14.8 & ab \\
Estudiar carrera técnica & 119 & 65.4 & 12.9 & $\mathrm{bc}$ \\
Estudiar carrera universitaria & 230 & 66.6 & 13.2 & $\mathrm{~cd}$ \\
Total & 474 & 64.6 & 13.9 & \\
letras diferentes indican que las diferencias entre las medias del nivel de expectativa son estadísticamente significativas al .05
\end{tabular}

\section{Evidencias de validez concurrente}

El FG de resiliencia de la escala CD-RISC mostró una correlación positiva de .452 , significativa $(p<.01)$ y en la dirección esperada con la medición del FG de la escala de resiliencia del CYRM-28 (Ungar \& Liebenberg, 2011). Esto demuestra validez concurrente moderada (FríasNavarro, 2015), pero aceptable.

\section{Evidencias de validez discriminante}

Considerando la variable expectativa de vida de la ficha sociodemográfica y las puntuaciones del factor general de la escala CD-RISC, los resultados del ANOVA fueron estadísticamente significativos $(\mathrm{F}=6.23,3$ g. de $1 ., p<.001)$. El estadístico de Levene fue de .4 $(p=.75)$, las pruebas post hoc se muestran en la Tabla 5, donde letras iguales indica que no hay diferencias estadísticamente significativas al .05 respecto al promedio de recursos resilientes medidos por la CD-RISC y las expectativas reportadas por los adolescentes y letras diferentes indican que existe diferencias estadísticamente significativas al 05 . Además, es posible reportar un tamaño del efecto de .038, considerado pequeño (Frías-Navarro, 2015), y la potencia observada de .964. Estos resultados muestran que, en general, a mayor expectativa reportada por los adolescentes, en promedio, se esperaría obtener mayores recursos resilientes reportados por la escala CD-RISC. Esta variable aporta evidencia débil de validez discriminante, lo que sugiere una mayor exploración en futuras investigaciones.

\section{Discusión}

En la presente investigación se examinó el comportamiento del CD-RISC como herramienta de detección y tamizaje de recursos resilientes para un grupo de 520 adolescentes (13 a 19 años) de educación secundaria en Chile. El estudio de las propiedades psicométricas, demostró que el $\mathrm{CD}$ RISC es un instrumento fiable en la medición de resiliencia en adolescentes escolarizados chilenos. Resultó de especial interés verificar en este estudio, la unidimensional en la estructura factorial del CDRISC, con un factor general de resiliencia, que integra todos los ítems del instrumento, reconociendo una respuesta resiliente global durante la adolescencia y con índices robustos y confiables. Esta unidimensionalidad había sido reportada para población universitaria española por García-León et al. (2019) y Chilena (Ponce, 2015). El modelo Bifactor permitió reconocer la unidimensionalidad global de resiliencia, no así los dos factores específicos que evidenciaron coeficientes omega jerárquicos de las subescalas, porcentaje de varianza relativa, porcentaje de varianza relativa de la subescala, varianza común explicada y replicabilidad de constructo, muy por debajo de los valores de corte, por lo que no se considera que poseen interpretación sustantiva al margen del factor general.

Sin embargo, la unidimensionalidad de la escala difiere con lo propuesto por los autores originales quienes determinan la presencia de cinco factores (Connor \& Davidson, 2003). Esta variabilidad en la estructura interna según 
población estudiada podría demostrar lo dinámico y adaptativo del constructo de resiliencia.

Los resultados obtenidos en esta población adolescente escolarizada no coinciden con los modelos de AFC verificados en población adulta expuesta a experiencias estresantes, donde se reconocen un modelo de 3 factores (ManzanoGarcía \& Ayala Calvo, 2013; Mealer et al., 2016; Xie et al., 2016), una hipótesis posible puede ser la variabilidad de recursos resilientes que se pueden ir desarrollando en grupos adultos más especializados y recurrentemente expuestos a montos altos de estrés, como lo son, las enfermeras de cuidados intensivos, soldados en China y los empresarios, configurando variables latentes diferenciadas.

En cuanto a la profundización en las evidencias de validez de estructura interna, mediante los diferentes modelos de invarianza evaluados, los resultados muestran que el instrumento puede ser aplicado tanto a hombres como a mujeres, no observando diferencias estadísticamente significativas en las medias latentes estimadas del factor general de resiliencia. Mismo resultado se evidenció considerando la variable edad.

Consiguientemente se demostró validez convergente moderada con una correlación significativa entre CD-RISC y CYRM-28 (Candia \& Blanco, 2019) en su versión adaptada en adolescentes chilenos, el tamaño de efecto fue moderado (Frías-Navarro, 2015) y esto puede responder a que la variable resiliencia medida por CYRM-28, implica evaluar respuestas resilientes en múltiples dimensiones incorporando la percepción de diferentes contextos para el adolescente. Bravo-Sanzana et al. (2015) y SalvoGarrido, San Martín, et al., (2020) reconocen la resiliencia como un mecanismo protector para el desarrollo integral del ser humano y la promoción de mayores expectativas de vida, estos hallazgos demuestran que a mayores expectativas (proyectos o metas) serían mayores los recursos resilientes de los adolescentes. Al contrario, cuando se observan bajas expectativas futuras habría una relación negativa con una relación negativa asociada a bajas expectativas y menores recursos resilientes de los adolescentes escolarizados chilenos.
Se recomienda, para estudios posteriores con adolescentes escolarizados, analizar el comportamiento de los ítems 2 y 3 del factor general de resiliencia que presentaron saturaciones factoriales estandarizadas inferiores a .3, pero estadísticamente significativos. Este resultado coincide parcialmente, ítem 3 , con el estudio de Manzano-García y Ayala Calvo (2013) realizado en población adulta expuesta a experiencias estresantes.

Este estudio también posee algunas limitaciones como es el tipo de muestra utilizada, no probabilística, adolescentes escolarizados en establecimiento que no corresponden a sectores de alta vulnerabilidad social, por tanto, este grupo podría estar menos expuestos a experiencias adversas, se sugiere replicar el estudio en grupos de adolescentes con mayor deserción escolar, adolescente en sistemas educativos para adultos y adolescentes con conductas desadaptivas y sancionados por el sistema penal de justicia.

\section{Referencias}

Abad, F. J., Olea, J., \& Ponsoda Gil, Vicente García, C. G. (2011). Medición en ciencias sociales y de la salud. Madrid: Síntesis.

Abad, F. J., Olea, J., \& Ponsoda Gil, Vicente García, C. G. (2011). Medición en ciencias sociales y de la salud. Madrid: Síntesis.

Aburn, G., Gott, M., \& Hoare, K. (2016). What is resilience? An integrative review of the empirical literature. Journal of Advanced Nursing, $\quad 72(5), \quad 980-1000$. https://doi.org/10.1111/jan.12888

Acevedo, V., \& Restrepo, L. (2012). De profesores, familias y estudiantes: Fortalecimiento de la resiliencia en la escuela. Revista Latinoamericana de Ciencias Sociales, Niñez y Juventud, 10, 301-319. http://revistaumanizales.cinde.org.co/index.ph $\mathrm{p} /$ Revista-

Latinoamericana/article/view/608/330

Alarcón, P., Wenger, L., Chesta, S., \& Salvo, S. (2012). Validez predictiva del instrumento Evaluación de Riesgos y Recursos (FER-R) para la intervención en adolescentes infractores de ley: Estudio preliminar* Predictive Validity at the Instrument Risk and 
Resources (FER-R) for Intervention Assessment with Youn. Universitas Psychologica, 11(4), 1657-9267.

Arias González, V. B., Crespo Sierra, M. T., Arias Martínez, B., Martínez-Molina, A., \& Ponce, F. P. (2015). An in-depth psychometric analysis of the Connor-Davidson Resilience Scale: Calibration with Rasch-Andrich model. Health and Quality of Life Outcomes, 13(1). https://doi.org/10.1186/s12955-015-0345-y

Artuch, R. (2014). Resiliencia y autorregulación en jóvenes navarros en riesgo de exclusión social, que acuden a programas de cualificación profesional inicial [Navarra]. http://dadun.unav.edu/handle/10171/36188

Ato, M., \& López, J. J. (2013). Un sistema de clasificación de los diseños de investigación en psicología. Anales de Psicología, 29(3), 1038-1059.

Barcelata, B. E. (2015). Adolescentes en riesgo. Una mirada a partir de la Resiliencia (S. Viveros Fuentes (Ed.); El Manual).

Barudy, J. (2015). Trauma y resiliencia: La resiliencia infantil en contextos de desigualdades sociales y de género. In $\mathrm{E}$. Saavedra, G. Salas, C. Cornejo, \& P. Morales (Eds.), Resiliencia y calidad de vida. La Psicología Educacional en diálogo con otras disciplinas (1ra ed., p. 202). Universidad Católica del Maule.

Bobes, J., Bascarán, M. T., Garcia-Portilla, M. P., Bousoño, M., Sáiz, P. A., \& Wallance, D. H. (2001). Banco de instrumentos básicos para la práctica de la psiquiatría clínica (Psiquiatría (Ed.); Segunda).

Bobes, J., Bascarán, M. T., Garcia-Portilla, M. P., Bousoño, M., Sáiz, P. A., \& Wallance, D. H. (2008). Banco de instrumentos básicos de psiquiatría clínica (Psiquiatría (Ed.)).

Bonifay, W. E., Reise, S. P., Scheines, R., Meijer, R. R., Bonifay, W. E., Reise, S. P., Scheines, R., \& Meijer, R. R. (2015). When are multidimensional data unidimensional enough for structural equation modeling? An Evaluation of the DETECT Multidimensionality Index. Structural Equation Modeling: A Multidisciplinary Journal, 1-13. https://doi.org/10.1080/10705511.2014.938596 Bonomelli, F., Castillo, A., \& Croquevielle, J.
(2020). Medición de la exclusión escolar en Chile (p. 93). Ministerio de Educación, Chile. https://centroestudios.mineduc.cl/wpcontent/uploads/sites/100/2020/04/DOCUME NTO-DE-TRABAJO-20_2020_f01.pdf

Bravo-Sanzana, M., Salvo-Garrido, S., Mieres, M., Mansilla, J., \& Hederich, C. (2017). Perfiles de desempeño académico: La importancia de las expectativas familiares. Perfiles Latinoamericanos, 25(50), 361-386. https://doi.org/10.18504/pl2550-016-2017

Bravo-Sanzana, M., Salvo-Garrido, S., \& Muñoz, C. (2015). Profiles of Chilean students according to academic performance in mathematics: An exploratory study using classification trees and random forests. Studies in Educational Evaluation, 44, 50-59. https://doi.org/10.1016/j.stueduc.2015.01.002

Brunner, M., Nagy, G., \& Wilhelm, O. (2012). A Tutorial on hierarchically structured constructs. Journal of Personality, 80(4), 796846.

https://doi.org/10.1111/j.14676494.2011.00749.x

Candia, S., \& Blanco, K. (2019). Validación de la Escala Child and Youth Resilience Measure (CYRM) en adolescentes escolarizados de Chile. Universidad de La Frontera.

Connor, K., \& Davidson, J. (2003). Development of a new Resilience scale: The ConnorDavidson Resilience scale (CD-RISC). Depression and Anxiety, 18(2), 76-82. https://doi.org/10.1002/da.10113

Cosco, T. D., Kaushal, A., Richards, M., Kuh, D., \& Stafford, M. (2016). Resilience measurement in later life: A systematic review and psychometric analysis. Health and Quality of Life Outcomes, 1-6. https://doi.org/10.1186/s12955-016-0418-6

Donnon, T., \& Hammond, W. (2007). A psychometric assessment of the self-reported Youth Resiliency: Assessing Developmental Strengths questionnaire. Psychological Reports, 100, 963-978. https://doi.org/10.2466/PR0.100.3.963-978

Dray, J., Bowman, J., Campbell, E., Freund, M., Wolfenden, L., Hodder, R. K., McElwaine, K., Tremain, D., Bartlem, K., Bailey, J., Small, T., Palazzi, K., Oldmeadow, C., \& Wiggers, J. (2017). Systematic review of 
universal resilience-focused interventions targeting child and adolescent mental health in the school setting. Journal of the American Academy of Child and Adolescent Psychiatry, 56(10), 813-824. https://doi.org/10.1016/j.jaac.2017.07.780

Farsen, T., Bogoni, A., \& Silva, N. (2017). Concepciones históricas y teoréticas sobre resiliencia en el trabajo: Una revisión integrativa de la literatura sobre el tema. Perspectivas En Psicología, 14(2), 18-29.

Ferrando, P. J., \& Lorenzo-seva, U. (2017). Assessing the quality and appropriateness of factor solutions and factor score estimates in exploratory item factor analysis. Educational and Psychological Measurement, 1-19. https://doi.org/10.1177/0013164417719308

Finney, S. J; DiStefano, C. (2006). Non-normal and Categorical data in structural equation modeling. In G. r. Hancock \& R. O. Mueller (Hrsg.). Struc- tural equation modeling: A second course (S. 269-314). Greenwich, Connecti- cut: Information age publishing. Structural Equation Modeling: A Second Course, 269-314.

Freiberg, A., Stover, J., de la Iglesia, G., \& Fernández, M. (2013). Correlaciones policóricas y tetracóricas en estudios factoriales. Ciencias Psicológicas, 21(2), 151164.

Frías-Navarro, D. (2015). Diseño y análisis en psicología aplicada - tamaño del efecto. Universitat de València. https://www.uv.es/ friasnav/efecto.html

Friborg, O., Hjemdal, O., Rosenvinge, J. H., \& Martinussen, M. (2003). A new rating scale for adult resilience: What are the central protective resources behind healthy adjustment? International Journal of Methods in Psychiatric Research, 12(2), 65-76. https://doi.org/10.1002/mpr.143

García-León, M. Á., González-Gómez, A., Robles-Ortega, H., Padilla, J. L., \& PeraltaRamírez, M. I. (2019). Propiedades psicométricas de la Escala de Resiliencia de Connor y Davidson (CD-RISC) en población española. Anales de Psicologia, 35(1), 33-40. https://doi.org/10.6018/analesps.35.1.31411

Hjemdal, O., Friborg, O., Stiles, T., \& Martinussen, M. (2006). A New Scale for
Adolescent Resilience: Grasping the central protective resources behind healthy development. Measurement and Evaluation in Counseling and Development, 39, 84-96. https://doi.org/10.1080/07481756.2006.11909 791

Hodder, R. K., Freund, M., Wolfenden, L., Dray, J., Kingsland, M., Lin, S., \& Wiggers, J. (2017). Systematic review of universal school-based 'resilience' interventions targeting adolescent tobacco, alcohol or illicit substance use: A meta-analysis. Preventive Medicine.

https://doi.org/10.1016/j.ypmed.2017.04.003

Jones, L. (2019). Resilience isn' $t$ the same for all: Comparing subjective and objective approaches to resilience measurement. WIREs Climate Change Published, October 2017, 119. https://doi.org/10.1002/wcc.552

Kline, R. B. (2005). Principles and practices od structural equation modeling (2nd ed.). Guilford.

Kueh, Y. C., Abdullah, N., Kuan, G., Morris, T., $\&$ Naing, N. N. (2018). Testing measurement and factor structure invariance of the Physical Activity and Leisure Motivation Scale for youth across gender. Frontiers in Psychology, 9(JUL), 1-12.

https://doi.org/10.3389/fpsyg.2018.01096

Lemos, V., Krumm, G., Gutierrez, M., \& Aránfilippetti, V. (2016). Desarrollo de una escala para evaluar recursos de personalidad asociados a la resiliencia infantil. Acción Psicológica, 13(2), 101-116. https://doi.org/10.5944/ap.13.2.17821

Li, C. R., Toland, M. D., \& Usher, E. L. (2016). Dimensionality, scoring, and interpretation of the Short Grit Scale.

Liebenberg, L., Ungar, M., \& van de Vijver, F. (2012). Validation of the Child and Youth Resilience Measure-28 (CYRM-28) among Canadian youth. Research on Social Work Practice, 22(2), 219-226. https://doi.org/10.1177/1049731511428619

Lorenzo-Seva, U., \& Ferrando, P. J. (2020). FACTOR (10.10.03). Universitat Rovira i Virgili. http://psico.fcep.urv.cat/utilitats/factor/

Luthar, S. S., Cicchetti, D., \& Becker, B. (2000). The construct of resilience: A critical 
evaluation and guidelines for future work. Child Development, 71(3), 543-562. https://doi.org/10.1111/1467-8624.00164

Manzano-García, G., \& Ayala Calvo, J. C. (2013). Propiedades psicométricas de la Escala de Resiliencia de Connor-Davidson en una muestra de emprendedores españoles. Psicothema, 25(2), 245-251. https://doi.org/10.7334/psicothema2012.183

Marsh, H. W., Hau, K., \& Wen, Z. (2004). In search of golden rules: Comment on hypothesis- testing approaches to setting cutoff values for fit indexes and dangers in overgeneralizing $\mathrm{Hu}$ and Bentler's (1999) Findings. Structural Equation Modeling, 11(3), 320-341.

Mealer, M., Schmiege, S. J., \& Meek, P. (2016). The Connor-Davidson resilience scale in critical care nurses: A psychometric analysis. Journal of Nursing Measurement, 24(1), 2839. https://doi.org/10.1891/1061-3749.24.1.28

Muthen, B. O. (2013). Measurement invariance with multigroups Retrieved. http://www.statmodel.com/discussion/messag es/9/11980.html?1456792993

Muthen, \& Muthen. (2019). Mplus (8.4). Copyrigh (C) 1988-2019.

Pinto-Cortez, C. G. (2014). Psychological resilience: An approach to the concept, theoretical framework and relation with child sexual abuse. Summa Psicológica UST, 11(2), 19-33.

https://dialnet.unirioja.es/servlet/articulo?codi go=4953998\&info=resumen $\&$ idioma $=$ SPA

Ponce, F. (2015). Análisis exploratorio de modelos de ecuaciones estructurales sobre la escala de resiliencia de Connor y Davidson (CD-RISC) en Chile y España. Salud \& Sociedad, 6(3), 238-247. https://doi.org/10.22199/s07187475.2015.000 3.00004

Reise, S. P. (2012). The Rediscovery of Bifactor Measurement Models. Multivariate Behavioral Research, 47(5), 667-696. https://doi.org/10.1080/00273171.2012.715555

Reise, S. P., Scheines, R., Widaman, K. F., \& Haviland, M. G. (2012). Multidimensionality and structural coefficient bias in structural equation modeling: A bifactor perspective. Educational and Psychological, 73(1), 5-26. https://doi.org/10.1177/0013164412449831

Rios, J., \& Wells, C. (2014). Validity evidence based on internal structure. Psicothema, 26(1), 108-116.

Rodríguez-Fernández, A., Ramos-Díaz, E., Ros, I., Fernández-Zabala, A., \& Revuelta, L. (2016a). Bienestar subjetivo en la adolescencia: El papel de la resiliencia, El autoconcepto y el apoyo social percibido. Suma Psicologica, 23(1), 60-69. https://doi.org/10.1016/j.sumpsi.2016.02.002

Rodríguez-Fernández, A., Ramos-Díaz, E., Ros, I., Fernández-Zabala, A., \& Revuelta, L. (2016b). Resiliencia e implicación escolar en función del sexo y del nivel educativo en educación secundaria. Aula Abierta, 44(2), 7782. https://doi.org/10.1016/j.aula.2015.09.001

Rodriguez, A., Reise, S. P., \& Haviland, M. G. (2015). Applying bifactor statistical indices in the evaluation of psychological measures. Journal of Personality Assessment. https://doi.org/10.1080/00223891.2015.10892 49

Rodríquez-Fernández, A., Ramos-Díaz, E., Ros, I., \& Zuazagoitia, A. (2018). Implicación escolar de estudiantes de secundaria: La influencia de la resiliencia, el autoconcepto y el apoyo social percibido. Educación XX1, 21(1), 87-108.

https://doi.org/10.5944/educXX1.16026

Roque Herńandez, M. del P., Acle Tomasini, G., \& García Méndez, M. (2009). Escala de resiliencia materna: Un estudio de validación en una muestra de madres con niños especiales. Revista Iberoamericana de Diagnóstico y Evaluación - e Avaliação Psicológica, 1(27), 107-132.

Ruiz Fernández, B., Gómez-Vela, M., Fernández Pulido, R., \& Badia Corbella, M. (2014). Determinantes de la calidad de vida del alumnado de primaria. Revista Iberoamericana de Educación, 66, 59-74. https://doi.org/10.35362/rie660378

Saavedra, E. (2010). Perfil de resiliencia en jóvenes de un liceo de alta vulnerabilidad de la séptima región. UCMAule Revista Académica, 38, 99-118.

Salvo-Garrido, S., Miranda-Vargas, H., VivalloUrra, O., Gálvez-Nieto, J. L., \& MirandaZapata, E. (2020). Estudiantes resilientes en el 
área de matemática: Examinando los factores protectores y de riesgo en un país emergente. Revista Iberoamericana de Diagnóstico y Evaluación - e Avaliação Psicológica, 2(55), 43-57. https://doi.org/10.21865/RIDEP55.2.04

Salvo-Garrido, S., Miranda Vargas, H., Vivallo Urra, O., Gálvez-Nieto, J., \& Miranda-Zapata, E. (2019). Students with socioeconomic disadvantages who have academic success in language: Examining academic resilience in South America. Preprints (Www.Preprints.Org), December, 1-11. https://doi.org/10.20944/preprints201912.004 3.v1

Salvo-Garrido, S., San Martín, S., Acuña-Cabrera, J., Vivallo-Urra, O., \& Gálvez-Nieto, J. (2020). Escuelas afectivas y sus estudiantes resilientes: $L a$ voz de las comunidades socioeducativas (S. Salvo-Garrido (Ed.); 1st ed.). Universidad de La Frontera.

Serrano, M., Garrido, M., Notario, B., Bartolomé, R., Solera, M., \& Martinez, V. (2012). Validez de la escala de Resiliencia de ConnorDavidson ( CD-RISC ) en una población de mayores entre 60 y 75 años. International Journal of Psychological Research, 49-57.

Smits, I. A., Timmerman, Marieke E., Barelds, D. P. H., \& Meijer, R. R. (2015). The Dutch Symptom Checklist-90-Revised is the use of the subscales justified? European Journal of Psychological Assessment, 31(4). https://doi.org/10.1027/1015-5759/a000233

Ten Berge, J. M. F., \& Kiers, H. A. L. (1991). A numerical approach to the approximate and the exact minimum rank of a covariance matrix. Psychometrika, 56(2), 309-315. https://doi.org/10.1007/BF02294464

Timmerman, M. E., \& Lorenzo-Seva, U. (2011). Dimensionality assessment of ordered polytomous items with parallel analysis. Psychological Methods, 16(2), 209-220. https://doi.org/10.1037/a0023353

Trizano-Hermosilla, I., \& Alvarado, J. M. (2016). Best alternatives to Cronbach's alpha reliability in realistic conditions: Congeneric and asymmetrical measurements. Frontiers in Psychology, 7(MAY).

https://doi.org/10.3389/fpsyg.2016.00769

Ungar, M., \& Liebenberg, L. (2011). Assessing resilience across cultures using mixed methods: Construction of the Child and youth resilience measure. Journal of Mixed Methods Research, 5(2), 126-149. https://doi.org/10.1177/1558689811400607

Vandenberg, R. J., \& Lance, C. E. (2000). A Review and synthesis of the measurement invariance literature: Suggestions, practices, and recommendations for organizational research. Organizational Research Methods, 3(4), 1-70. https://doi.org/10.1177/109442810031002

Villalta, M., Delgado, A., Escurra, L., \& Torres, W. (2017). Resiliencia y rendimiento escolar en adolescentes de Lima y de Santiago de Chile de sectores vulnerables. Universitas Psychologica, 16(4), 1-10. https://doi.org/10.11144/Javeriana.upsy164.rrea

Wagnild, G. M., \& Young, H. M. (1993). Development and psychometric evaluation of the Resilience Scale. Journal of Nursing Measurement, 1(2), 165-178.

West, S. G., Taylor, A. B., \& Wu, W. (2012). Model fit and model selection in structural equation modeling. In Handbook of structural equation modeling (pp. 209-231). R. H. Hoyle.

Windle, G., Bennett, K., \& Noyes, J. (2011). A methodological review of resilience measuremengregory bateson ecology mindt scales. Health and Quality of Life Outcomes, 9(8), 1-18. https://doi.org/10.1186/1477-7525-9-8

Xie, Y., Peng, L., Zuo, X., \& Li, M. (2016). The psychometric evaluation of the ConnorDavidson resilience scale using a Chinese military sample. PLoS ONE, 11(2), 1-10. https://doi.org/10.1371/journal.pone.0148843

Yu, C., \& Muthen, B. (2002). Evaluation of model fit indices for latent variable models with categorical and continuous outcomes. Annual Meeting of the American Educational Research Association.

https://www.statmodel.com/download/Yudiss ertation.pdf

Zinbarg, R. E. (2006). Estimating generalizability to a latent variable common to all of a scale's indicators: A comparison of estimators for $\omega \mathrm{h}$. Applied Psychological Measurement, 30(2), 121-144. https://doi.org/10.1177/0146621605278814 